Noman 2016, 34(2), 77-86

Revista de Psicologia, Ciències de l’Educació i de l'Esport

ISSN: 1138-3194

Copyright (c) 2016

www.revistaaloma.net

\title{
El nuevo fetichismo del internet de las cosas en la sociedad kidult
}

\author{
Glòria Callicó ${ }^{1} \&$ Jordi Celma ${ }^{2}$ \\ 1 Universidad de Barcelona \\ ${ }^{2}$ Universidad Autónoma de Barcelona
}

Recibido: 17-4-2016

Aceptado: 15-9-2016

\section{El nuevo fetichismo del internet de las cosas en la sociedad kidult}

Resumen. Este trabajo estudia la influencia futura del internet de las cosas, objetos conectados a internet para, teóricamente, mejorar nuestras vidas, al igual que su impacto en nuestro modo de vivir y la emergencia de nuevas psicopatologías. El internet de las cosas cambiará cada una de nuestras vidas; en algunos aspectos, en un sentido positivo, pero, por otro lado, también incrementará la incidencia de psicopatologías como el TDAH, la anorexia, la bulimia, la depresión, y las adiciones a los videojuegos, especialmente en la generación kidult. Los kidults, adultos que aspiran a comportarse como niños, quieren evadir sus temores de envejecer, y algunos aparatos del internet de las cosas relacionados con la atención médica y la salud pueden proveer un soporte efectivo contra esos temores, pero pueden también funcionar como fetiche tecnológico y, a la larga, aumentar su inmadurez. Reflexionaremos sobre el impacto futuro del internet de las cosas, su relación con la fetichización tecnológica y el incremento de determinadas patologías.

Palabras clave: internet de las cosas; generación kidult; fetiche tecnológico; TDAH; adición a los videojuegos

\section{The new fetishism of the Internet of Things in a society of «kidults»}

Summary. This paper studies the future influence of the Internet of Things, defined as physical objects connected to the Internet such that they theoretically improve our lives, and it examines their impact on our way of life and the emergence of new psychological pathologies. The Internet of Things will change all of our lives; in some ways this will be for the better, the phenomenon will also increase the incidence of pathologies such as ADHD, anorexia, bulimia, depression, and addiction to video games, especially in the 'kidult' generation. Kidults, or adults that aspire to behave like kids, want to evade their fear of ageing, and some devices arising from the Internet of Things related to healthcare and fitness can represent an effective buffer against this fear, but they can also function as a technological fetish and, in the long term, increase users' immaturity. We will reflect on the future impact of the Internet of things, its relation with technology fetishism and the increase in the prevalence of certain pathologies.

Key words: Internet of Things; Kidult generation; technology fetishism; ADHD; video game addiction 
Cualquier tecnología suficientemente avanzada es indistinguible de la magia

Arthur C. Clarke

\section{Introducción}

En este artículo trataremos del internet de las cosas para referirnos a los objetos conectados vía internet que nos ofrecen información, y, a su vez, nos servirá para que tomemos decisiones en un determinado ámbito de nuestra vida, y apuntaremos cómo modificará sensiblemente nuestro futuro antes de cinco años.

Se estima que en 2020 habrá más de seis dispositivos conectados por persona. Actualmente, en las paradas de autobús de una smart city como Barcelona, ya podemos saber el tiempo de espera previsto; en un futuro próximo, estas aplicaciones se irán generalizando, afectarán a la industria, por ejemplo, a los coches que compremos y, en general, a la vida cotidiana y a nuestra salud y calidad de vida. Algunos hospitales ya están empezando a implementar habitaciones «inteligentes» que permitirán monitorizar las constantes vitales de los pacientes, volcar la información en su historial médico, programar futuras pautas de control y reducir las tareas rutinarias del personal y evitar errores de transcripción de los datos obtenidos.

Esta es una primera etapa pero, en una sociedad cada vez más envejecida y con los gastos sanitarios disparados, el paso siguiente será sustituir controles y pruebas en las que ahora intervienen profesionales médicos y que exigen un desplazamiento físico a ambulatorios por controles efectuados por aparatos interconectados instalados en nuestro hogar o implantados en nuestro cuerpo.

En una sociedad tan centrada en el culto al cuerpo y tan individualista como la actual, los dispositivos portables o implantables serán fácilmente aceptados e, incluso, deseados, porque permitirán individualizar la medicina y empoderar a los pacientes, haciéndoles sentir que ellos tienen el control para conseguir sus propios objetivos de salud y de calidad de vida.

De acuerdo con el The World Agrees: Technology Inspires Optimism for Healthcare (Intel Newsroom, 2016), más de la mitad de las personas encuestadas confiaría más en un test personalizado y supervisado por ellos mismos que en uno estándar realizado por un profesional médico. Más del 70\% también se muestra dispuesto a utilizar dispositivos y sensores en su hogar y a seguir las correspondientes prescripciones. Todo ello puede llevar a que el propio paciente acabe interpretando sus datos y sustituya la opinión del médico.

Utilizaremos el concepto de fetichismo de la tecnología como una metodología de análisis, que permite razonar por qué en diversos contextos se sobreestima la tecnología más allá de la utilidad específica para la cual ha sido diseñada por sus creadores. Está fetichización puede darse a distintos niveles, hay tecnologías que solo están al alcance de los estados, por ejemplo la militar o la que se aplica a grandes infraestructuras; para muchos gobernantes disponer de armas de destrucción masiva, que con un mínimo sentido común no utilizarán nunca, les permite alimentar su egolatría; para otros países, las obras públicas tienen un valor simbólico más allá del uso concreto de la misma, por eso se desea construir el puente más largo, el rascacielos más alto o tener, como España, más kilómetros de AVE que cualquier otro país europeo.

El fetichismo de la tecnología se refuerza y populariza aún más cuando la tecnología está al alcance de la mayoría de los ciudadanos y sirve para cosas concretas de nuestra vida cotidiana. Además potencia el narcisismo porque nos permite ser en muchos casos co-creadores de nuestra propia realidad.

Este fetichismo tiene muchas posibilidades de seguir aumentando en el futuro, a medida que se incremente la penetración de internet y se añadan nuevas funciones a los objetos actuales, porque será muy útil para los estados neoliberales, que han recortado sanguinariamente el estado del bienestar y pretenden seguir haciéndolo en el área de la educación y de la salud; incorporando tecnología que sustituya la mayor cantidad posible de profesionales, bajo el pretexto de que en ningún caso disminuye la calidad del servicio, aunque parte del trabajo que hoy hacen los profesionales interpretando datos facilitados por las máquinas deberá ser efectuada por los propios pacientes, tomando decisiones sin, muchas veces, estar preparados, aunque su ego les sugiera lo contrario.

Esta fetichización probablemente será aún más fácil por las especiales características de unos ciudadanos que constituyen la denominada sociedad kidult, a medio camino entre los jóvenes (kids) y los adultos (adults), compradores compulsivos con una mentalidad en parte infantil y para los cuales estos instrumentos interconectados y que conocen solo muy superficialmente son una prolongación de sí mismos y un placebo que camufla sus déficits y sus ansiedades.

El objetivo de este trabajo es reflexionar sobre el impacto del internet de las cosas en nuestras vidas, analizar las consecuencias sociales de esta nueva fetichización donde se sobrestima la tecnología más allá de la utilización para la cual ha sido creada y apuntar nuevas patologías o el incremento de otras ya existentes fruto de este funcionamiento social.

\section{La generación kidult}

En la actualidad, las fronteras generacionales son mucho más difusas que a finales del siglo pasado. Los clásicos indicadores del paso de los jóvenes a la edad adulta se han ido diluyendo en la sociedad posmoderna y es difícil concluir cuándo se alcanza realmente la madurez.

En el siglo pasado, los indicadores más habituales eran la finalización del periodo de educación formal, la consecución de una independencia económica y una vivienda independiente a la de los padres, y también, en muchos casos, el matrimonio y la construcción de una nueva familia. 
Hoy en día, todo resulta menos definido porque la formación nunca acaba realmente y nos habremos de actualizar durante toda la vida; la precariedad económica hace que los jóvenes no sean totalmente independientes hasta edades avanzadas y, en ocasiones, se produzca un efecto boomerang, ya que parejas de jóvenes van a vivir juntos hasta que se rompe la relación $\mathrm{y}$, como sus ingresos son escasos, vuelven temporalmente a casa de los padres hasta el momento de iniciar otro ciclo de ida y vuelta.

Así, el adulto, como prototipo de estabilidad, experiencia, madurez, autoconfianza e integración en los valores de su comunidad, frente a la inseguridad, el individualismo y el egocentrismo del joven aún inmaduro, se ha quebrado, al menos parcialmente. Estamos viviendo en una sociedad cada vez más inmadura, que no se compromete y que no cumple lo prometido, tal como comenta Gary Cross (2010).

La juventud era una etapa cronológica de la vida de las personas, por eso George Bernard Shaw dijo: «la juventud es una enfermedad que se cura con los años», pero, en la actualidad, tal como comenta Bernardini (2014), ser joven ya no es una situación transitoria, sino una opción, una forma de vida, bien establecida y brutalmente promovida por los medios de comunicación.

Los primeros que observaron la existencia creciente de adultos que consumían como jóvenes y niños fueron los cazadores de tendencias y expertos de marketing, que acuñaron el concepto de kidult, mezcla de kid y de adult; adultos que quieren conservar al niño que fueron, pero no como un recuerdo, sino comprando y consumiendo con las pautas propias de los niños, aunque con mayor poder adquisitivo.

Los kidult pueden ser perfectamente racionales en muchas de sus decisiones, sin embargo, pueden gastar sumas elevadas, en ocasiones por encima de sus posibilidades reales, para atender sus gustos y caprichos, su deseo de diversión y de ocio ha de satisfacerse de inmediato y parecen tener capacidad de dinero ilimitada, aunque hayan de comprar a crédito productos que en absoluto son de primera necesidad.

Un kidult emblemático podría ser Michael Jackson, que aseguraba que no quería crecer y que, gracias a su gran poder adquisitivo, vivía en una mansión parecida a un parque temático y tenía unos comportamientos que podemos calificar, como mínimo, de excéntricos.

Observando la realidad que nos rodea encontramos por todos lados rasgos de una sociedad kidult, personas que permanentemente juegan a videojuegos, leen comics, ven dibujos animados, practican paintball, coleccionan juguetes antiguos, hacen colas inmensas para comprarse el último iPad.

Los kidults compran, casi a cualquier precio, productos que los retrotraigan a la infancia y sostengan sus recuerdos y vivencias; a la vez que también buscan nuevas experiencias siempre que sean lúdicas, divertidas y de sensación inmediata. En la actualidad, hay estrategias de marketing dirigidas específicamente al segmento kidult con el continuo lanzamiento de pro- ductos que antes se consideraban solo para niños y jóvenes. Por lo tanto, hay un refuerzo mediático permanente de estas pautas de consumo, que, obviamente, inciden también en pautas de comportamiento personal más profundo.

Limitarnos a analizar las pautas de consumo de los kidults, como hacen los cazadores de tendencias, podría ser útil para un análisis de marketing, pero eso sería centrarse en una visión evidente y muy superficial; sin ir al núcleo de la cuestión: ¿por qué los kidults se comportan como jóvenes inmaduros? O bien preguntarnos: ¿kidult es simplemente una forma de definir a un segmento de mercado o es una psicopatología aún por estudiar?

\section{Los kidults en la sociedad de consumo}

Conseguir transformarse en un kidult es relativamente sencillo; según Bonazzi y Pusceddu (2008), para capturar esta estación de la vida, la juventud permanente, es suficiente con querer modificar el propio cuerpo y la propia mente interrumpiendo las relaciones consolidadas, reinventándose día a día.

Esa reinvención casi diaria se correlaciona con otros rasgos que analiza Bernardini (2012, 2013), y que básicamente se observan también en los niños y jóvenes. La impetuosidad frente a la supuesta reflexividad de los adultos; la dependencia excesiva de los demás frente a una mayor independencia; la duda constante y la búsqueda de la satisfacción instantánea frente a los objetivos a largo plazo; la egocentricidad y el narcisismo frente a la sociabilidad, la indefinición de la identidad sexual o bisexualismo, la no aceptación de diferencias entre jóvenes y adultos, y el sentirse con derecho a todo sin ninguna obligación respecto a los demás.

Esa reinvención pretende conducir a una recompensa inmediata que sacie, aunque sea sólo puntualmente, su ego; y que no dependa de la relación con los demás ni cuestione su narcisismo, y sólo puede conseguirse mediante la gratificación del juego o del consumo.

Frente al homo sapiens o al homo faber, en la actualidad, nos encontramos ante el homo ludens, ya anticipado por Huizinga (2012) o el homo consumer de Baudrillard (1998), o en la mayoría de los casos una combinación de ambos.

Como razona Todd (2012), pocas cosas juegan un papel tan importante en nuestras vidas como el consumo. El consumo está íntimamente ligado a la creación y producción del propio yo. Los productos tienen un significado que va más allá de su propia función primaria, y el consumo es la forma cotidiana (y en muchos casos la única) del consumidor para comunicarse con la sociedad que le rodea. De acuerdo con Baudrillard, nuestras compras reflejan nuestros deseos más profundos y nuestro consumo está ligado a nuestra personalidad y nuestras carencias, confundiendo el tener con el ser, buscando una identidad a través de adquirir y consumir (Fromm, 2007). 
El consumo no es sólo (que lo es) un sistema económico dominante en la actualidad; es la forma en que nuestra sociedad funciona y la forma en que interactúan sus ciudadanos, los consumidores. Nuestro consumo nos ayuda a proyectar nuestra imagen frente a los demás y, a su vez, nos facilita las herramientas para convertirnos, ni que sea puntualmente, como en aquel histórico programa de la televisión española, Reina por un día (que consistía en conceder durante un día los sueños de la mujer elegida en cada programa), en quienes queramos ser. Por eso razonamos que el consumo nos permite reinventarnos, mostrarnos y vendernos día a día. Las redes sociales, Facebook, Twitter y otras páginas web, facilitan ese mostrarnos y vendernos a los demás.

De modo que, a mayor capacidad de consumo, mayor proyección social. Ese es uno de los argumentos (el otro es su conservadurismo ideológico) del candidato a la presidencia de Estados Unidos, Donald Trump. Trump exhibe su músculo financiero cuando dice que no necesita recaudar fondos porque es millonario, sus credenciales son sus millones, sus jets privados, sus mansiones, sus casinos, nunca sus estudios u otras capacidades intelectuales. Trump está diciendo: «soy importante porque puedo comprar todo esto... y si he podido amasar tanto dinero seré un buen presidente para vosotros».

Pero el consumismo va más allá de la mera asociación de la capacidad de compra como indicador del éxito social. Lo que compramos es aquello con lo que fantaseamos y lo que fantaseamos es aquello de lo que carecemos, la parte de nosotros mismos que está castrada, que sentimos que nos falta, aunque lo ignoremos.

Baudrillard (1998) ya anticipó la transición de una sociedad ascética, organizada alrededor del trabajo y sacrificio, hacia una sociedad dirigida a la búsqueda del disfrute, no importa la forma de conseguirlo. En nuestra sociedad resulta casi obligatorio ser feliz, McGowan (2013) comenta que hemos pasado del concepto de la «obligación» al de la «obligación de disfrutar»y es precisamente esa orden, la que nos transmite el superyó. Un superyó insaciable poco diferenciado del ello, esa parte más ligada a lo instintivo y a lo impulsivo, que ya no encarna la prohibición y que no conduce a un comportamiento decente y al sacrificio, sino al mandato social del capitalismo de disfrutar sin límite $y$, si esto no se consigue, hace sentir culpable al individuo; en estos momentos el hecho de sentirse culpable por no lograr disfrutar y ser feliz puede ser incluso un motivo de consulta psicológica.

Esa obligación de disfrutar día a día solo se consigue efímeramente mediante el juego o el consumo, pero, si al día siguiente no se puede volver a experimentar, surge una frustración porque más allá de ese corto plazo no se ha construido nada más.

Tal como indica Barber (2008), el sistema neoliberal capitalista convierte a los ciudadanos en consumidores, pero más que dejarles realmente la opción de escoger qué consumir los acaba consumiendo a ellos. Porque cuanto más hipnotizado está el ciudadano con el con- sumo y más infantil es su comportamiento, más fuerte será la fetichización que tendrá con ciertos productos.

\section{El fetichismo tecnológico}

Entendemos por fetiche un objeto al cual, algunas personas y en ciertos contextos, le atribuyen propiedades y significados especiales distintos de sus propiedades y características objetivas, y que se utiliza para protegernos de la ansiedad que nos causa una carencia.

El fetichismo ha sido utilizado inicialmente en tres campos: el del análisis religioso, en el económico (Marx) y en el de la sexualidad (Freud); pero, en general, el fetichismo, como instrumento metodológico de análisis, tal como razonó Appadurai (1986), puede utilizarse siempre que estudiemos apariencias superficiales que enmascaran significados subyacentes $u$ ocultos.

Más recientemente, autores como Marenko (2014) proponen utilizar como metodología de análisis un neoanimismo, con un significado análogo al fetichismo.

La idea de que los objetos y otras entidades no humanas tienen un alma, una vida propia y características humanas es evidente cuando nos fijamos en cómo hablamos a nuestros ordenadores, coches y smartphone, y esperamos que nos contesten. Sin duda, se está creando un animismo digital o tecnológico, y está relación irá en aumento a medida que se implemente el internet de las cosas.

Que las cosas «tienen vida y propiedades diversas», al menos algunas cosas para algunas personas, es evidente para los más de tres millones de personas que han comprado el libro de Kondo (2015), que explica cómo ordenar tu hogar; porque tal como dice su autora, transformando tu casa en un espacio limpio y ordenado de manera permanente verás sorprendido cómo cambia tu vida. Es impensable creer que tres millones de personas lean a Kondo simplemente por la aparente función del libro de poner orden en su casa.

Probablemente, los lectores y seguidores de esta autora lo que quieran es cambiar su vida, pero confunden su casa con su mente y, evidentemente, es mucho más fácil reordenar la casa y ver qué falta o qué nos sobra que plantearnos un nivel mucho más profundo de cambio, por ejemplo, reflexionando y valorando qué nos aporta o nos deja de aportar una relación determinada y si vale la pena seguir conservándola. En este sentido, la casa y el orden en las cosas funcionaría como fetiche.

Está socialmente aceptado que la tecnología ha cambiado nuestras vidas y observamos día a día que lo seguirá haciendo en el futuro, esta capacidad de cambio permanente induce, como indica Huggett (2004), a un fetichismo de lo nuevo. Así, citando palabras del propio De Kerckhove (1997): «cuando aparece una tecnología que amplía una de nuestras facultades y trasciende nuestras limitaciones físicas, ansiamos adquirirla y utilizarla al máximo»; es decir, que la nueva tecnología crea su propia demanda. Así, las continuas actualiza- 
ciones de las aplicaciones que descargamos en nuestro móvil acaban saturando el espacio disponible para esas descargas, lo que nos empuja a comprar otro móvil más rápido y con mayor capacidad.

En ocasiones, una tecnología pensada para una finalidad específica, como los drones que fueron creados para ejecutar sin ningún amparo legal a supuestos terroristas, se populariza para otros fines comerciales o simplemente lúdicos y crea una demanda ingente como si de un juguete se tratara. En una sociedad kidult, el fetichismo de lo nuevo es evidente.

Pero como cada vez la tecnología es más compleja y sus fabricantes desean vender a más y más clientes, aunque sus conocimientos tecnológicos sean virtualmente nulos, se hace preciso crear interfaces que faciliten el uso, aun cuando se desconozca el funcionamiento real del objeto. Se pasa, pues, de saber cómo funciona un objeto a observar simplemente los resultados, a veces solo aparentes, del funcionamiento del mismo.

Entonces nos encontramos ante lo que Huggett (2004) denomina «el fetichismo de la máscara». Esta opacidad, en muchos casos, es deliberada, porque cuanto menos sepamos cómo funciona realmente el objeto, más fácil será vendernos un substituto que supuestamente mejore al anterior.

En adelante, cuando hablemos de tecnología, nos referiremos a la fundamentada en la informática e internet, las que coloquialmente se denominan «nuevas tecnologías», ya que en la actualidad no tiene sentido que cualquier avance científico no se sustente parcial o totalmente en ellas. Permanentemente aparecen mejoras ligadas a conceptos como más rapidez, mayor información disponible, mayor resolución, mayor autonomía, etc., que seguirán manteniendo y aumentando el fetichismo de estas tecnologías.

\section{Internet de las cosas: fetichismo a la carta}

Internet de las cosas va a potenciar, sin duda, una profundización y una mayor generalización del uso de internet, tanto en el número de usuarios como en el número de aplicaciones y utilidades de esos usuarios. Habrá perfiles de usuarios distintos, pero ahora nos centramos en el del ciudadano kidult, proconsumidor, apresurado en sus decisiones, individualista, narcisista y con necesidad de gratificación inmediata y permanente.

El aforismo The medium is the Message, que introdujo Marshall McLuham (1994), sirve para razonar que cualquier tecnología (por ejemplo, internet) es a la vez extensión y amputación o anulación del ser humano. McLuham lo ejemplificaba diciendo que un martillo es una extensión del brazo y que nos permite hacer cosas que antes no podíamos, pero que si solo utilizamos el martillo, nos hará perder algunas de nuestras habilidades.

McLuham critica esa pérdida de capacidad genérica, pero más recientemente otros autores, como Nicholas Carr (2011), ya inciden en la pérdida de capacidades concretas, como la falta de concentración y la reducción del sentido crítico de las personas, atribuible en su opinión, al uso masivo de internet y a lo que él llama «surfeo» sobre los textos o lectura diagonal. Este mismo autor cita un comunicado de la Administración Federal de Aviación de EE. UU. en el que se alerta de que los pilotos de aviones se están volviendo demasiado dependientes de los pilotos automáticos y otros sistema informatizados, y acaba con la recomendación de que los pilotos pasen más tiempo volando manualmente para evitar la degradación de la capacidad de estos para volar.

En nuestra opinión, y volviendo al ejemplo de McLuham, creemos que internet de las cosas realzará aún más esa extensión, porque el martillo conectado a internet será martillo y diez cosas más, y nos informará sobre la orientación y la intensidad de la fuerza a realizar para conseguir un determinado efecto. Además, como el mundo será cada vez más complejo, la ignorancia relativa de todos nosotros respecto al conjunto del saber generado será cada vez mayor y tenderemos a confiar cada vez más en una tecnología que solo conoceremos a nivel de usuario.

Como decía Bertrand Russell: «una de las dificultades importantes del mundo es que los ignorantes están completamente seguros y los inteligentes están llenos de dudas». Hoy en día nada ha cambiado, pero con la debilitación del estado protector, el enaltecimiento del individualismo y el advenimiento de internet, muchas personas se han envalentonado unas a otras mediante la redes sociales y toman todo tipo de decisiones que ponen en riesgo su salud, por ejemplo, deciden que no es necesario vacunarse o vacunar a sus hijos, o arriesgan su patrimonio, invirtiendo en productos derivados de alto riesgo financiero.

Todo esto ha debilitado la jerarquía tradicional, de manera que el médico puede ser ahora una información no contrastada en un post de internet, el maestro un pseudocurso gratuito en una plataforma cualquiera y el psicoterapeuta un minilibro de autoayuda.

Cada vez será más frecuente el efecto de Dunning y Kruger (1999), que concluyeron que: «la sobrevaloración del incompetente nace de la mala interpretación de la capacidad de uno mismo y la infravaloración del competente nace de la mala interpretación de la capacidad de los demás».

Este sesgo cognitivo hace que las personas con pocas habilidades o conocimientos tengan una sensación ilusoria de que son mucho más competentes de lo que realmente son, debido a su propia incompetencia que les imposibilita reconocer sus propios errores y aceptar la competencia de los que están mejor preparados que ellos.

Actualmente es usual observar que algunas personas sin suficiente competencia en una materia hacen razonamientos inadecuados y que, fruto de esa incompetencia, encuentran justificaciones espurias que les impidan darse cuenta de sus verdaderos errores. Como botón de muestra, simplemente basta observar el incremento del número de rescates en excursiones por 
la montaña de personas que se han aventurado sin preparación y equipo, intentando emular a un prodigio de facultades como Kilian Jornet.

Además, hay aspectos externos a los propios usuarios de internet, como pueden ser las propias actuaciones de los organismos públicos, que pueden potenciar un mayor uso de internet de las cosas y una mayor asunción de una autoconfianza excesiva que haga que el usuario magnifique ese instrumento conectado a internet, que le aconseja y le ayuda a tomar decisiones, aunque no sean las más adecuadas.

Como hemos comentado en la introducción, en un futuro próximo serán las propias autoridades sanitarias, con intención de reducir costes, las que intenten sustituir al personal sanitario por instrumentos conectados que puedan realizar ciertas actividades de supervisión de la salud; finalmente, eso conducirá a delegar en los propios usuarios las tareas de control e interpretación de resultados de los instrumentos ubicados en sus domicilios, al margen de que se hayan financiado o no con recursos públicos.

Ese mayor uso de aparatos conectados a Internet aumentará el sesgo de autoconfianza de los usuarios; todos nosotros facilitaremos información propia sobre nuestras pautas de consumo, comportamiento y datos biológicos, y el instrumento nos irá aconsejando qué hacer en cada caso y qué productos hemos de consumir o qué servicios hemos de contratar para ser eternamente jóvenes y estar eternamente saludables.

En consecuencia, nos encontraremos ante un fetichismo a la carta y una dependencia absoluta del «brujo» (el Gran Hermano que controlará todas nuestras conexiones vía internet, con cookies y otros dispositivos, y que gestionará esa información desde un servidor ubicado en la nube), que nos aconsejará qué hacer en cada momento y nos venderá los productos adecuados en cada etapa de nuestra vida.

\section{Internet de las cosas: los seres humanos en el bucle}

No existe en la actualidad una investigación consolidada sobre las consecuencias que en el futuro pueda producir la generalización del internet de las cosas, pero existe un creciente interés sobre este aspecto. Desde 2014, el Institute of Electrical and Electronic Engineers publica una revista científica sobre el tema, la IEEE Internet of Things Journal, donde se analizan especialmente problemas tecnológicos inherentes al internet de las cosas.

En un artículo de la citada revista Stankovic (2014) resume las futuras áreas de investigación en el internet de las cosas, y, a pesar de centrarse en aspectos tecnológicos, dedica un apartado de su artículo a analizar un nuevo concepto que él define como: «Humans in the loop», para referirse a la situación futura en la cual los humanos se encontrarán en el centro de un bucle de ordenadores con los que tendrán que relacionarse de una forma distinta a la que lo han hecho hasta ahora.

Los humanos se relacionarán de una forma sinérgica con los ordenadores, recibiendo información que analizarán para tomar decisiones que, a su vez, serán nuevos inputs para el sistema de ordenadores con el que se relacionan. Stankovic comenta que, aunque la interacción de humanos y computadoras será beneficiosa en muchos aspectos, como la seguridad viaria o la atención sanitaria, se plantea el desafío de cómo este proceso acabará modelando el comportamiento humano, en especial en el complejo aspecto fisiológico y psicológico. Stankovic concluye que internet de las cosas seguirá cambiando nuestra forma de vida, como ya lo han hecho Facebook, Twitter o WhatsApp, pero que la forma cómo lo harán es actualmente impredecible.

Sin duda, siguiendo en razonamiento de McLuham (1994), internet de las cosas, como cualquier tecnología, es una especie de prótesis del ser humano. Pero esta prótesis básicamente sirve para transaccionar información entre máquinas y humanos. El concepto de Transactive Memory es una hipótesis psicológica (Wegner, Giuliano \& Hertel, 1985) para referirse a un mecanismo a través del cual grupos de personas codifican, almacenan, comparten y recuperan, cuando lo precisan, un determinado conjunto de conocimientos. Inicialmente, la memoria transactiva fue estudiada a nivel de parejas y de familias, pero, posteriormente, su uso se ha extendido a grupos y organizaciones que acaban compartiendo un sistema de memoria común; memoria o cultura común es un concepto que se utiliza frecuentemente a nivel de empresas, corporaciones o grupos generacionales.

En la actualidad podemos generalizar aún más y considerar que internet, que entre otras cosas es el repositorio más grande de información que existe en el mundo, es la base de transacción de memoria que más utilizamos actualmente los humanos.

Hay que precisar que en internet cualquiera puede publicar, en una web, un blog o en un foro; en consecuencia, la información que obtenemos en la actualidad no es neutra, es decir, que en general no somos bastante conscientes de que puede no ser fidedigna o realmente representativa de la realidad; en muchos casos, la atribuimos falsamente a nuestro propio conocimiento (Fisher, Goddu \& Keil, 2015) y así incrementa nuestra confianza en las deducciones o decisiones que tomamos basándonos en nuestras propias opiniones y en la información obtenida de internet.

Podemos concluir que nuestras decisiones se fundamentan, cada vez más, en nuestra memoria interna y en la memoria transactiva que en vez de proceder de padres, tutores o colegas procede, en mayor medida, de internet. En cierto modo, (Fisher et al., 2015) nuestras mentes consideran que internet es como una memoria transactiva que amplía el conocimiento al cual tenemos acceso, ya que, en muchos casos, tras un tiempo de búsqueda, nuestro cerebro olvida que la información se obtuvo de internet y la asocia directamente a la propia memoria interna.

Otro aspecto relacionado con internet que no podemos obviar es el exceso de seguridad que, en general, se concede a la información obtenida mediante esa 
tecnología. El concepto de overconfidence ha sido ampliamente utilizado en psicología desde los años sesenta, pero es tan amplio que puede ser definido de diferentes formas. Moore y Healy (2008) analizan tres significados distintos para ese concepto: la sobreestimación de los propios resultados obtenidos, la mejor ubicación relativa de uno mismo respecto a terceros (better-than-average), y el exceso de fiabilidad o de confianza en las propias opiniones o creencias. El artículo citado reconcilia las distintas formas de exceso de seguridad y razona cómo aparecen con más frecuencia unas u otras según el tipo de actividad que se contrasta. Pero en ningún caso se asocia una u otra forma de exceso de seguridad a una mayor o menor utilización de internet en el proceso.

Con posterioridad se ha analizado el concepto de overconfidence relacionado con otras áreas del conocimiento, en concreto en el de las finanzas y las inversiones; y muy en particular en las inversiones en renta variable y en operaciones del mercado de divisas, (coloquialmente conocido como Forex).

Es sabido que, en el campo de las inversiones, disponer de la información relevante de las empresas antes que los competidores supone una ventaja comparativa importante a la hora de obtener beneficios, por lo tanto, interesa disponer de la máxima información viable y fiable en el menor tiempo posible. Así, en el campo de las finanzas, el concepto de overconfidence puede plantear una definición un poco distinta de las tres usadas tradicionalmente en el campo psicológico.

Skala (2008), cuando se refiere al exceso de confianza en el campo de las finanzas, define este concepto (overconfidence in finance) como la sobreestimación del propio conocimiento o de la precisión de la información de la cual se dispone, o de la interpretación de la misma. Surge, por lo tanto, una nueva acepción del concepto de overconfidence fundamentada en la sobrevaloración del volumen o la calidad de la información de la cual se dispone para tomar decisiones. Esta nueva acepción está relacionada con la utilización masiva de internet como repositorio para consultar información sobre cualquier actividad o decisión de deseemos emprender.

Como internet de las cosas amplifica la relación entre los computadores y las personas a todos los niveles, laboralmente, en el cuidado y la salud, en la domótica, en la conducción asistida, necesariamente aumenta la información que fluye entre computadores y humanos y, lógicamente, disminuye la proporción de información que fluye solo entre humanos.

Surgen, pues, dudas acerca de los efectos que puede tener está nueva «relación» entre humanos y computadoras a medida que internet de las cosas sigue incrementando su presencia en nuestras vidas, podemos intuir que internet de las cosas será un inmenso repositorio de información, que habrá que controlar para evitar riesgos de privacidad y delitos económicos o utilización abusiva de esa información, pero también deberían analizarse otros riesgos que pudieran afectar a la salud de los usuarios, desde la perspectiva psicológica.
Como ejemplo, basta recordar que los profesionales, que trabajamos en la educación superior y tenemos que tutorizar trabajos final de grado, podemos constatar fácilmente cómo curso tras curso aumenta el porcentaje de bibliografía obtenida a través de internet y, generalmente, con poco criterio de selección en detrimento de otras fuentes de información impresas en papel.

Podemos intuir que algunos trastornos ya existentes aumentarán en el futuro; por ejemplo, el hecho de estar cada vez más interrelacionados con máquinas que sustituirán a persona, puede producir que la depresión vaya en aumento, debido a mayor situación de soledad que experimentarán, principalmente, las personas mayores que cada vez más estarán atendidas por aparatos tecnológicos y quizás por sus familiares o médicos, pero, en muchas ocasiones, vía online, por cual necesariamente se reducirá su vida social.

También pueden aumentar los trastornos asociados a los celos y los temores paranoicos, debido a la facilidad de poder controlar a otras personas, por ejemplo, a través de GPS instalados en el móvil que, en estos momentos, ya nos permiten saber el lugar exacto donde se encuentra una persona. Parejas que controlarán a sus cónyuges, padres que controlarán permanentemente a sus hijos adolescentes. Mensajes que nos avisarán del importe que hayamos gastado y del saldo que nos quede en la cuenta corriente. Pulseras que nos computarán los pasos que demos cada día, las calorías que gastemos, conduciéndonos a un aumento de la obsesión por el culto al cuerpo y quitándonos libertad y espontaneidad. Es evidente que todo ello puede generar gran malestar e incidir en que emerjan psicopatologías aun no imaginadas.

Estas psicopatologías requerirán, en muchas ocasiones, ayuda psicológica. Entender cada caso por sí mismo en su individualidad, pero sin olvidar que el proceso se encuentra enmarcado en el contexto social posmoderno y neoliberal en que aparecen nos ayudará a entender mejor el significado del trastorno y tratarlo.

\section{Psicopatologías emergentes}

Fruto del funcionamiento social que hemos descrito al hablar de la generación kidult, asociado a la necesidad de estar siempre conectado, a la búsqueda permanente de información, al apresuramiento y al consumismo que apenas sirve para paliar fútilmente nuestras carencias, y del uso cada vez más frecuente de internet como instrumento de «surfeo», y como repositorio de nuestra memoria externa utilizada sin análisis crítico, han aparecido algunas psicopatologías nuevas a la vez que ha aumentado el número de casos de otras ya existentes y que amenazan en convertirse en una epidemia.

El TDAH, trastorno por déficit de atención con hiperactividad, es uno de estos trastornos que ha aumentado espectacularmente en estos últimos años. En una época en que todos vivimos acelerados y en que se premia la rapidez que proporcionan las nuevas tec- 
nologías (Ubieto, 2014), el diagnóstico de TDAH está creciendo desmesuradamente. Una mayoría de niños son ahora etiquetados con este diagnóstico, pero quizás es esta sociedad posmoderna la que sin ser consciente está fabricando «niños TDAH» en la medida en que no les enseñamos a respetar, a aplazar la satisfacción, mientras que aumenta la permisividad y la laxitud en las normas.

La aceleración con la que nos enfrentamos al día a día provoca dispersión y puede ser una de las causas de las dificultades para centrar la atención. Vivimos en un mundo en el que se piensa poco, y de un modo superficial. Un mundo que confunde los sentimientos con las sensaciones, y en el que se proponen pocos objetivos más allá de los que se pueden conseguir a corto plazo y con poco esfuerzo. El esfuerzo ya no está de moda, todo lo que supone estar sujeto a una disciplina se ha desvalorizado en beneficio del culto al deseo y de su realización inmediata. Podríamos pensar que la falta de atención de los alumnos es similar al funcionamiento de un telespectador, excitado e indiferente a la vez, y sobresaturado de informaciones.

Otra psicopatología emergente es el TLP, trastorno límite de la personalidad propio de la adolescencia, caracterizado también por la poca tolerancia a la frustración, la necesidad de satisfacción inmediata de los impulsos, un yo frágil con falta de límites, la falta de autocontrol y una inestabilidad en las relaciones. Probablemente, el incremento de este trastorno está en relación con la dificultad de poner límites de algunos padres que confunden autoridad con autoritarismo y que, como en el caso de los niños TDAH, no ayudan a que el niño pueda ser capaz de postergar los deseos y no le ofrecen un modelo de contención necesario para conseguir una adecuada estabilidad (Guimón, 2013).

Otros trastornos como la bulimia, a veces alternado con la anorexia, son trastornos alimentarios que, principalmente, afectan a las mujeres que plasman las carencias y las necesidades en la comida, de forma que la persona puede comer compulsivamente sin ser consciente de que el vacío que está intentando llenar no es el de su estómago, sino el afectivo. Los nuevos cambios del rol de la mujer, sin duda, tienen un papel importante, tal como explican Maganto y Cruz (2000), por una parte, la mujer lucha por encontrar un equilibrio entre los nuevos ideales de éxito: ser autosuficiente, competente, ambiciosa, con éxito y esbelta, ya que la delgadez suele asociarse al éxito y, por otra parte, con una preocupación por el aspecto y la imagen corporal que va en aumento, relacionado con el deseo de mantenerse joven, dinámica y atractiva, pero detrás de la cual hay un mercado de moda masivo y consumista. Toda esta multiplicidad de exigencias de roles conduce a una crisis de identidad de la mujer que pueden precipitar tanto la bulimia como la anorexia.

Crecen las adicciones a los videojuegos, principalmente de los videojuegos en línea que, según apuntan algunos estudios, son los que más provocan adicción o un uso problemático, tal como prefieren algunos autores denominarlo (Carbonell, Fuster, Chamarro \&
Oberst, 2012). Aunque Young (1998) ya empezó a estudiar los signos de alarma que nos pueden indicar cuándo se ha atravesado el umbral de la afición para pasar a la adicción, hay que destacar cierta controversia en torno a si realmente se puede hablar de adicción -término frecuentemente usado para referirnos a una sustancia química que causa dependencia fisiológica y una afectación a nivel del sistema nervioso- o es mejor emplear el término de uso problemático o desadaptativo de las nuevas tecnologías (Carbonell \& Oberst, 2015).

En este sentido, los juegos interactivos en los que se escoge un avatar en que se crea y modifica tanto la apariencia física como la personalidad a voluntad resultan especialmente atractivos para los jóvenes, porque uno puede convertirse en un personaje admirado y respetado, y puede ser todo lo contrario de lo que siente que es en su vida real. En estos entornos, el premio es siempre ser visible, recibir muchas visitas, obtener el reconocimiento y la atención de personas desconocidas potencialmente idealizadas en el contexto de una interacción anónima y despersonalizada donde la identidad que despliegan en la red está totalmente bajo nuestro control. Mantener la reputación virtual y mantener los lazos sociales creados puede incidir en que uno vuelva a conectarse una y otra vez tal como especifica Wallace (2001). La adicción en general a cualquiera de los medios de las redes sociales, Facebook, Twitter o WhatsApp, es un fenómeno que va en aumento y no solamente en la gente joven. Es habitual ver, en el metro o en el autobús, a prácticamente casi la totalidad del público conectada a través del móvil, estudiantes que en clase continuamente están haciendo uso de él y, en los gimnasios, hay usuarios que lo utilizan incluso durante las actividades dirigidas.

Otra polémica gira en torno a si los videojuegos violentos provocan mayor número de conductas violentas, lo que parece bastante demostrado según algunos estudios realizados (McLean y Griffiths, 2013). Así, por ejemplo, parece que hay una correlación significativa de una mayor aceptación de la agresión física y menor empatía. Nosotros pensamos que presenciar de manera asidua acciones violentas, sea en los juegos online o en cualquiera de los medios de comunicación, conduce a minimizar estas mismas acciones violentas. Otros estudios señalan correlaciones entre la afición excesiva a este tipo de juegos violentos y el un uso excesivo de ingesta de drogas, alcohol y una pobreza en las relaciones sociales y familiares (Padilla-Walker, Nelson, Carroll \& Jensen, 2009). Pero, posiblemente, no sea tan simple como que una es causa directa de la otra, sino que haya una tendencia predisponente a la adicción en muchos individuos tanto a cualquier tipo de substancias como a los juegos debido a sus notables carencias afectivas. No existe, tampoco, acuerdo en que este tipo de juego provoque también mayor depresión como apuntan algunos estudios (Ferguson, San Miguel \& Hartley, 2009), ya que nuestra experiencia clínica con adolescentes adictos a este tipo de juegos más bien 
nos demuestra todo lo contrario, pues son precisamente los adolescentes que se sienten deprimidos los que más tienden a refugiarse en este tipo de juego para canalizar, de este modo, su agresividad y frustración, tal como apuntarían también otros estudios citados en Pedrero, Rodríguez y Ruz (2012).

\section{Conclusiones}

La tecnología no tiene límites y la penetración de internet en nuestras vidas parece que tampoco. En los próximos años, muchos objetos que ahora ya utilizamos, y otros que aún están por producirse, incorporarán aplicaciones informáticas que ampliarán su utilidad para las personas que, en principio, libremente, quieran utilizarlas.

No obstante, en algunos ámbitos, nos veremos casi obligados a usarlas -como nos vemos obligados a disponer de una tarjeta de crédito o de un carné de conducir- fruto de la presión social, aunque no siempre nos guste.

Los alumnos, en la enseñanza, y los empleados y profesionales, en sus empresas y lugares de trabajo, cada vez utilizarán más internet, pero su uso no necesariamente reducirá su interacción con otras personas. Con los mayores, que ya padecen más soledad, la situación puede ser distinta; esas personas mayores, cuando intenten recibir servicios asistenciales, probablemente verán sustituidos a cuidadores y a acompañantes de carne y hueso por máquinas y robots que incrementarán el aislamiento.

En la mayoría de los casos, esas aplicaciones sustituirán tareas y servicios que ahora realizan otras personas y exigirán interactividad por parte del usuario, que, además, habrá de ser capaz de interpretar o ejecutar las instrucciones recibidas.

En un entorno marcadamente individualista y en una sociedad neoliberal, con un nivel de paro elevado y unos trabajadores con salarios cada vez más miserables, serán cada vez más frecuentes las sensaciones de soledad y angustia, y los instrumentos conectados a internet serán quienes, en muchos casos, ocupen ese vacío.

Para algunas personas esos instrumentos pueden acabar convirtiéndose en fetiches y sus usuarios en fetichistas tecnológicos, porque un fetichista es alguien que, aferrándose a su fetiche, puede soportar su situación actual. Y cuanto más descarnada sea la realidad, más necesario será el fetiche.

Ese fetichismo puede acabar produciendo consecuencias negativas. Aumentará la sensación de muchas personas, con un perfil narcisista y un exceso de autoconfianza en sus conocimientos y capacidades, de dominar una tecnología que realmente no conocen; lo que provocará que muchos usuarios sean víctimas de engaños y sufran abusos de tipo financiero, de explotación de su intimidad y de fraudes de todo tipo; $y$, en consecuencia, pueden aparecer figuras delictivas hoy desconocidas, nuevas psicopatologías y aumentar otras ya existentes asociadas al estrés de las nuevas situaciones.
Sin llegar a esos extremos podemos observar que, en muchos casos, internet se ha convertido en el mayor repositorio de información que utilizan los humanos, en detrimento de otras fuentes de memoria externa que se han usado tradicionalmente. Esa utilización, a veces abusiva, de la información obtenida de internet ya está produciendo un sesgo de exceso de confianza en cuanto a la calidad, cantidad y veracidad de la información utilizada, que finalmente conduce a la toma de decisiones precipitadas o erróneas que pueden aumentar la frustración o afectar la autoestima de los afectados.

No podemos concretar el efecto adicional que producirá el advenimiento del internet de las cosas, ya que aún no hay estudios empíricos, pero sería razonable pensar que, como mínimo, exacerbará las patologías existentes al sustituir, en cierta medida, la relación entre humanos por una mayor interrelación computador-humano-computador. Eso sitúa a los humanos en un nuevo bucle con consecuencias hoy por hoy impredecibles.

Sin duda, en el futuro se tendrá que seguir profundizando en los efectos que la cada vez mayor interrelación entre humanos y computadores tendrá para la relación social y los problemas de salud mental derivados de la nueva situación.

\section{Referencias}

Appadurai, A. (1986). The Social Life of Things: Commodities in Cultural Perspective. Cambridge: Cambridge University Press.

Baudrillard, J. (1998). The Consumer Society: Myths and Structures. London: SAGE Publications.

Barber, B.R. (2008). Consumed: How Markets Corrupt Children, Infantilize Adults and Swallow Citizens Whole. New York: Norton.

Bernardini, J. (2012). Adulti nel tempo dell'eterna giovenezza. Milán: Franco Angeli

Bernardini, J. (2013). Adulti di carta. La rappresentazione sociale dell'età adulta sulla stampa. Milán: FrancoAngeli.

Bernardini, J. (2014). The infantilization of the Postmodern Adult and the Figure of Kidult. Postmodern Openings, 5(2). 39-55. http://dx.doi.org/10.18662/ po/2014.0502.03

Bonazzi, F.\& Pusceddu, D. (2008). Giovani per sempre. La figura dell'adulto nella posmodernità. Milán: FrancoAngeli.

Carbonell, X., Fuster, H., Chamarro, A. \& Oberst, U. (2012). Adicción a internet y móvil: una revisión de estudios empíricos españoles. Papeles Del Psicólogo, 33(2), 82-89.

Carbonell, X. \& Oberst, U. (2015) Las redes sociales en línea no son adictivas. Aloma, 33(2), 13-19.

Carr, N. (2011). ¿Qué está haciendo Internet con nuestras mentes? Superficiales. Madrid: Taurus.

Cross, G. (2010). Men to Boys. The making of modern immaturity. New York: Columbia University Press.

De Kerckhove, D. (1997). The Skin of Culture: Investigating the New Electronic Reality. London: Kegan Page. 
Dunning, D. \& Kruger, J. (1999). Unskilled and Unaware of it: How Difficulties in Recognizing One's Own Incompetence Lead to Inflated Self-Assessments. Journal of Personality and Social Psychology, 77(6), 1121-1134.

Fisher, M., Goddu, M.K. \& Keil, F.C. (2015). Searching for Explanations: How the Internet Inflates Estimates of Internal Knowledge. Journal of Experimental Psychology, 144(3), 674-687. http://dx.doi.org/10.1037/ xge0000070

Ferguson, C.J., San Miguel, S. \& Hartley, T. (2009). Multivariate analysis of youth violence and aggression: The influence of family, peer, depression and media violence. Journal of Paediatrics, 155, 904-908. Fromm, E. (2007) Del tener al ser. Barcelona: Paidos Ibérica. Guimón, J. (2013). Estado actual de la psicoterapia basada en la transferencia (PBT) de Kernberg. Avances en Salud Mental Relacional, 12(1). Available at: http:// hdl.handle.net/10401/6196 [Accessed 12 Aug. 2016].

Huggett, J. (2004). Archaeology and the New Technological Fetishism. Archeologia e Calcolatori, 15, 81-92.

Huizinga, J. (2012). Homo Ludens. Madrid: Alianza Editorial.

Intel Newsroom. (2016). The World Agrees: Technology Inspires Optimism for Healthcare. Intel Newsroom. Available at: https://newsroom.intel.com/news-releases/the-world-agrees-technology-inspires-optimism-for-healthcare/ [Accessed 12 Aug. 2016].

Kondo, M. (2015). La Magia del orden. Madrid: Aguilar. McLean, L. \& Griffiths, M.D. (2013). The psychological effect of videogames on young people: A review. Aloma, 31(1), 119-133.

McLuham, M. (1994). Understanding Media. The extensions of Man. Cambridge: MIT Press.

Maganto, C. \& Cruz, S. (2000). La imagen corporal y los trastornos alimenticios: una cuestión de género. Sepypna. Cuadernos de psiquiatría y psicoterapia del niño $y$ del adolescente, 30, 45-48.

Marenko, B. (2014). Neo-Animism and Design. A New Paradigm in The Object Theory. Design and Culture, 6(2), 219-242. http://dx.doi.org/10.2752/17547081 4X14031924627185

McGowan, T. (2013). Enjoying what we don't have. The Political Project of Psychoanalysis. Lincoln: University of Nebraska Press.

Moore, D. \& Healy, PJ. (2008). The trouble with Overconfidence. Psychological Review, 115(2), 502-517. http://dx.doi.org/10.1037/0033-295X.115.2.502

Padilla-Walker, L.M., Nelson, L.J.,Carroll, J.S. \& Jensen, A.C. (2009). More than just a game: Video game and internet use during emerging adulthood. Journal of Youth And Adolescence, 39, 103-113.

Pedrero E.J., Rodríguez M.T. \& Ruz J.M. (2012). Adicción o abuso de teléfono móvil. Revisión de la literatura. Adicciones, 24(2), 139-152. Available at: http://www. redalyc.org/articulo.oa?id=289122912007 [Accessed 12 Aug. 2016].

Skala, D. (2008). Overconfidence in Psychology and Finance - An Interdisciplinary Literature Review. Bank i Kredyt. 4, 33-50. Available at: http://ssrn.com/abstract=1261907 [Accessed 12 Aug. 2016].

Stankovic, JA . (2014). Research Directions for the Internet of Things. IEE Internet of Things Journal 1(1), 3-9. http://dx.doi.org/10.1109/JIOT.2014.2312291

Todd, D. (2012). You Are What You Buy: Postmodern Consumerism and the Construction of self. Hohonu, $10,48-50$.

Ubieto, J.R., (2014). TDAH: hablar con el cuerpo. Barcelona: UOC.

Wallace, P. (2001). La psicología de Internet. Barcelona: Paidós Ibérica.

Wegner, D.M., Giuliano, T. \& Hertel, P.T. (1985). Cognitive interdependence in close relationships. In: W.J.Ickes (ed.), Compatible and incompatible relationships. New York: Springer Verlag.

Young, K. (1998). Internet Addiction: The emergence of a new clinical disorder. Cyberpsychology \& Behavior, 1, 237-244.

\section{El nou fetitxisme de la internet de les cosas} en la societat kidult

Resum. Aquest treball estudia la influència futura de la internet de les coses, objectes connectats a internet per a millorar, teòricament, les nostres vides, i el seu impacte en la manera de viure i l'emergència de noves psicopatologies. La internet de les coses canviarà cada una de les nostres vides; en alguns aspectes, en un sentit positiu, però, d'altra banda, també incrementarà la incidència de psicopatologies com el TDAH, l'anorèxia, la bulímia, la depressió, $i$ les addicions als videojocs, especialment en la generació kidult. Els kidults, adults que aspiren a comportar-se com a nens, volen evadir els temors d'envellir, $i$ alguns aparells de la internet de les coses relacionats amb l'atenció mèdica $i$ la salut poden proveir un suport efectiu contra aquests temors, però també poden funcionar com a fetitxe tecnològic $i$, a la llarga, augmentar-ne la immaduresa. Reflexionarem sobre l'impacte futur de la internet de les coses, la seva relació amb la fetitxització tecnològica i l'increment de determinades patologies.

Paraules clau: internet de les coses; generació kidult; fetitxe tecnològic; TDAH; addició als videojocs 